\title{
Male Circumcision in Our Society - A Paradigm Shift
}

\author{
Duncan D Mugala ${ }^{1}$, Stephen Mhango ${ }^{2}$, Chomba Sikasote ${ }^{3}$ \\ ${ }^{1}$ BSc, MBChB, MMed, FCS(ecsa). Senior Lecturer Copper Belt University-School Of Medicine, Consultant Surgeon Ndola Central \\ Hospital \& Konkola Copper Mines Plc Hospitals (Retired), Honorary Lecturer, University of Zambia School of Medicine. \\ ${ }^{2}$ BSc, MBChB, Registrar in Surgery Konkola Copper Mines.
}

${ }^{3}$ BSc HB, MB ChB, MMed, FCS (ECSA) Consultant Surgeon, Urologist: KCM Mine Hospitals Chingola.

\begin{abstract}
Circumcision is an operation as old as mankind, it is performed worldwide by many tribes and cultures as an initiation rite and it is routinely done at birth for a large number of boys in much of the English speaking world. In Zambia it has been practiced by a minority group of tribes from times immemorial often referred to as "Mukanda". In the recent past there has been a phenomenal rise in the numbers of people requesting to be circumcised. We looked at the requests for circumcision from the beginning of June 2007 to the end of May2008; we collected the reasons for the request, age and tribe of the requester. Circumcisions which were actually carried out, the time it took for the wound to completely heal and complications if any were noted. We also looked at the Operation records for circumcision from December 1999 to December 2003. A period of five years were also studied. There were 212 requests for circumcision in the year under review. The youngest was three months old the oldest was 47 years the mean age was 38.3 years. The main reason for requesting circumcision was for social reasons; The findings were $37 \%$ in children and $53 \%$ in adults. Of the 212 requests, $128(60.4 \%)$ were circumcised. 117(91.4\%) were fully healed by the end of two weeks. 11(8.6\%) patients had delayed healing. The thinking behind the social request was HIV infection prevention. We also noted that the request for circumcision cut across the tribal divide.
\end{abstract}

Keywords: Adults, Circumcision, Children, HIV

\section{Introduction}

Circumcision is an operation as old as mankind, highlighted today by Jewish and Muslim tradition in which all males are usually circumcised as part of their faith. However it is performed worldwide by many tribes and cultures as an initiation rite and it is routinely done at birth for a large number of boys in the USA, Australia, Canada, New Zealand, South Africa and much of the English speaking world. Overall it is estimated that a quarter of the male population is circumcised - about 750 million males. It is possible that circumcision arose independently in different cultures for different reasons. It has been variously proposed that male circumcision began as a religious sacrifice, a rite of passage, a form of sympathetic magic to ensure virility, a means of suppressing (or enhancing) sexual pleasure, an aid to hygiene, a means of marking those of lower (or higher) social status, as a tribal identity, a treatment for masturbation or other socially proscribed sexual behaviors etc [10]. The tomb artwork in Egypt thought to have been done between 2345 and 2181 BC is said to be the oldest documentary evidence of circumcision [10]. Throughout history it has been embraced and denounced at various times and by various peoples for example; ancient Greek aesthetics of the human form considered circumcision a mutilation of a previously perfectly shaped organ and the first-century Alexandrian Apion denounced circumcision as a barbaric custom. The Church issued a papal edict in 1442 that prohibited the practice of circumcision for all Christians. But routine neonatal circumcision was promoted during late Victorian times in the English-speaking parts of Canada, Australia, New Zealand, the United States and the United Kingdom and was widely practiced during the first part of the 20th century in these countries[10].
Circumcision in Zambia has been practiced from times immemorial often referred to as "Mukanda", this practice however was not carried out by all the tribes in Zambia in fact only a minority group of tribes practiced it.

In the recent past there has been a phenomenal rise in the numbers of people requesting to be circumcised. This has come because of the belief and scientific studies, that circumcision offers some protection against infection by the HIV: Zambians have come to learn that observational studies have suggested and described a geographical correlation between lower HIV prevalence and high rates of male circumcision in some countries in Africa and elsewhere. This has been demonstrated in three randomized controlled trials(RTCs) undertaken in Kisumu, Kenya, Rakai District, Uganda and Orange Farm, South Africa that male circumcision reduces the risk of heterosexually acquired HIV infection in men by approximately $60 \%$. As a result of such studies, the WHO/UNAIDS, in March 2007, convened an international consultation including policy makers, programme managers, researchers, and AIDS and women's health advocates to examine this issue and make policy and programme recommendations[1],[9]. Indeed some of the recommendations have led to media publicity hence causing a rise in demand for circumcision.

Where as in Zambia in the past requests for circumcision were mainly for traditional reasons and a few for medical reasons. We are seeing people requesting for circumcision for a larger number of reasons, These reasons which in the past would not have warranted a request for circumcision. We are also seeing the fact that the requests are coming from people from tribes which traditionally did not practice circumcision. The circumcising tribes are the Lundas, Luvales, Luchazis, Ndembus, and the Chokwes,in the North 


\section{International Journal of Science and Research (IJSR) \\ ISSN (Online): 2319-7064 \\ Index Copernicus Value (2013): 6.14 | Impact Factor (2015): 6.391}

western province of Zambia. In the Western province there are the Mbundas and the Nkoyas. In the Luapula province there are the Kazembe Lundas where the practice was by and large limited to the Royalty. The Muslim community also carry out circumcision as matter of religious requirement. The Majority of the 73 tribes in Zambia did not as a tradition carry out circumcision unless there were strong medical indications. This paper seeks to demonstrate that there has been a paradigm shift in our people's view of circumcision.

\subsection{Patients and Methods.}

\subsubsection{The prospective study}

We collected all the requests for circumcision from the beginning of June 2007 to the end of May 2008 noting the name, age, tribe and the reason for the request. We also recorded how many of the requests for circumcision were actually carried out . Of those who got circumcised we noted when the wound got completely healed and we took note of any complications.

\subsubsection{The retrospective study}

We also looked into the operation records from December 1999 to December 2003 a period of five years .Initially we wanted to compare just one year in the past but the numbers were so small that we decided five year period would give us enough retrospective data to study.

\section{Results}

\subsection{Age Distribution}

There were 212 requests for circumcision in the year under review. The youngest was three months old the oldest was 47 years the mean age was 38.3 years.

Table 1: Age Distribution

\begin{tabular}{|c|c|c|c|c|c|c|c|c|c|c|}
\hline $\begin{array}{c}\text { Age } \\
\text { range }\end{array}$ & $0-5$ & $6-10$ & $11-15$ & $16-20$ & $21-25$ & $26-30$ & $31-35$ & $36-40$ & $41-45$ & $46-50$ \\
\hline $\begin{array}{c}\text { No. of } \\
\text { patients }\end{array}$ & 40 & 23 & 32 & 14 & 24 & 36 & 22 & 11 & 8 & 2 \\
\hline
\end{tabular}

There were 212 requests for circumcision in the year under review. The youngest was three months old the oldest was 47 years the mean age was 38.3 years.

\subsection{The Requests for Circumcision}

There were several reasons advanced for the request of circumcision.

Table 2: Reasons for Circumcision Requests

\begin{tabular}{|c|c|c|c|}
\hline Reason & $\begin{array}{c}\text { In Children } \\
(\%)\end{array}$ & $\begin{array}{c}\text { In Adults } \\
(\%)\end{array}$ & $\begin{array}{c}\text { Patients } \\
(\%)\end{array}$ \\
\hline Balanitis & $01(1)$ & $02(2)$ & $03(2)$ \\
\hline Foreskin Sores & $00(0)$ & $31(27)$ & $31(15)$ \\
\hline Phymosis & $42(44)$ & $09(8)$ & $51(24)$ \\
\hline Religious Request & $01(1)$ & $04(3)$ & $05(3)$ \\
\hline Social Request & $35(37)$ & $62(53)$ & $97(46)$ \\
\hline Traditional Request & $16(17)$ & $06(5)$ & $22(10)$ \\
\hline Tight Phrenulum & $00(0)$ & $01(1)$ & $01(0.5)$ \\
\hline Warts & $00(0)$ & $02(2)$ & $02(1)$ \\
\hline Total & $95(100)$ & $117(100)$ & $212(100)$ \\
\hline
\end{tabular}

Table 3: Reason for Circumcision among the Tribes

\begin{tabular}{|c|c|c|c|c|c|}
\hline Tribe & Traditional & $\begin{array}{c}\text { Foreskin } \\
\text { Sores }\end{array}$ & $\begin{array}{c}\text { Social } \\
\text { Request }\end{array}$ & Phymosis & $\begin{array}{c}\text { Total } \\
(100 \%)\end{array}$ \\
\hline Bemba & 00 & 15 & 31 & 20 & 70 \\
\hline Bisa & 00 & 00 & 00 & 02 & 02 \\
\hline Lozi & 00 & 02 & 08 & 03 & 24 \\
\hline Lunda & 9 & 01 & 02 & 00 & 12 \\
\hline Luvale & 10 & 00 & 02 & 00 & 12 \\
\hline Lamba & 00 & 00 & 04 & 00 & 04 \\
\hline Lambya & 00 & 00 & 00 & 01 & 01 \\
\hline Lenje & 00 & 00 & 00 & 01 & 01 \\
\hline Kaonde & 02 & 03 & 01 & 04 & 10 \\
\hline Kazembe & 00 & 00 & 01 & 00 & 05 \\
\hline Kunda & 00 & 01 & 00 & 00 & 02 \\
\hline Lala & 00 & 01 & 06 & 03 & 10 \\
\hline Namwanga & 00 & 03 & 07 & 03 & 12 \\
\hline Ngoni & 00 & 01 & 05 & 00 & 06 \\
\hline Mambwe & 00 & 01 & 01 & 03 & 06 \\
\hline Tonga & 00 & 00 & 08 & 02 & 11 \\
\hline Tumbuka & 00 & 01 & 05 & 03 & 10 \\
\hline Chewa & 00 & 00 & 01 & 02 & 03 \\
\hline Nsenga & 00 & 01 & 04 & 00 & 05 \\
\hline Senga & 00 & 01 & 03 & 00 & 04 \\
\hline Tabwa & 00 & 00 & 01 & 00 & 01 \\
\hline Ushi & 00 & 01 & 01 & 01 & 03 \\
\hline Foreigners & 01 & 00 & 04 & 00 & 06 \\
\hline & & & & & \\
\hline
\end{tabular}

A survey was taken in Chingola getting the view of the Bemba people about Circumcision. The following we mate were; people who were workers in the mine company, Pastors from various Churches, Chingola residents Church leaders and the general Chingola residents. 20 male persons were asked simply: "would you allow a Bemba man to be circumcised? This was carried out in 2008 May. The questioned people were never told that Circumcision was actually going on in the hospital among their people. They gave their own opinion. They were Typical Bemba people. Fifteen told me that Circumcision can never be done among the Bemba people. Three were not so sure about it and two said circumcision is the best thing for everyone.

\subsection{Outcome of Circumcisions Carried Out}

There were 212 patients who requested for circumcision. 128(60.4\%) patients presented themselves for the circumcision. $117(91.4 \%)$ of the circumcised were fully healed by the end of two weeks. 11(8.6\%) patients had delayed healing. The reasons for the delayed healing were: Mild wound infection in 09(7.03) patients, one patient had severe wound infection. In one patient there was no reason why the wound healing was delayed. The other complications encountered were, Hematoma formation in one patient, postoperative bleeding in four patients and in one patient the sore in the foreskin turned out to be a carcinoma of the Penis

\subsection{The Retrospective Review}

There were 85 cases requiring circumcision during the five year period. These were distributed as follows: 


\section{International Journal of Science and Research (IJSR) ISSN (Online): 2319-7064 \\ Index Copernicus Value (2013): 6.14 | Impact Factor (2015): 6.391}

Table 7: Indication of Circumcision in Children

\begin{tabular}{|c|c|}
\hline Indication & Patients (\%) \\
\hline Phymosis & $24(38.7)$ \\
\hline Traditionl Request & $34(54.8)$ \\
\hline Paraphymosis & $04(6.5)$ \\
\hline Total & $62(100)$ \\
\hline
\end{tabular}

Dilatation of fore skin was done in 06 of children with Phymosis.18 had circumcision

Table 8: Indication for Circumcisin in Adults

\begin{tabular}{|c|c|}
\hline Indication & Patients (\%) \\
\hline Penile Warts & $04(17)$ \\
\hline Phymosis & $06(26)$ \\
\hline Social Requested & $13(57)$ \\
\hline Total & $23(100)$ \\
\hline
\end{tabular}

\subsection{Comparison between the Past and Now}

Table 9: Comparison Between the Past and Now

\begin{tabular}{|c|c|c|c|c|}
\hline Reason & $\begin{array}{c}\text { In Children } \\
(\%) \\
\text { Present }\end{array}$ & $\begin{array}{c}\text { In Children } \\
(\%) \\
\text { Past }\end{array}$ & $\begin{array}{c}\text { In Adults } \\
\text { Present }\end{array}$ & $\begin{array}{c}\text { In Adults } \\
\text { (\%) } \\
\text { Past }\end{array}$ \\
\hline Balanitis & $01(1)$ & 0 & $02(2)$ & 0 \\
\hline Foreskin Sores & $00(0)$ & 0 & $31(27)$ & 0 \\
\hline Phymosis & $42(44)$ & $24(38.7)$ & $09(8)$ & $06(26)$ \\
\hline Paraphymosis & 0 & $04(6.5)$ & 0 & 0 \\
\hline Religious Request & $01(1)$ & 0 & $04(3)$ & 0 \\
\hline Social Request & $35(37)$ & 0 & $62(53)$ & $13(57)$ \\
\hline Traditional Request & $16(17)$ & $34(54.8)$ & $06(5)$ & 0 \\
\hline Tight Phrenulum & $00(0)$ & 0 & $01(1)$ & 0 \\
\hline Warts & $00(0)$ & 0 & $02(2)$ & $04(17)$ \\
\hline Total & $95(100)$ & $62(100)$ & $117(100)$ & $23(100)$ \\
\hline
\end{tabular}

\subsection{Number of Reasons Presented for Circumcision}

In the recent study there were five reasons presented for the request of circumcision in children and eight in adults. In the past there were only three reasons presented in both children and adults

\section{Discussion and Conclusion}

Following the results of the African circumcision Randomized Controlled Trials and the approval of the WHO and UNAIDS whose recommendations emphasized that male circumcision is only partially protective and therefore should always be considered as part of a comprehensive HIV prevention package, which includes the provision of HIV testing and counseling services; treatment for sexually transmitted infections; the promotion of safer sex practices; the provision of male and female condoms and promotion of their correct and consistent usethere has been a mad rash in Africa to implement solution to the AIDS crisis in subSaharan Africa[1],[9]. Countries such as Rwanda and Uganda have pushed forward plans for mass circumcision despite clear problems with both the trials and the implementation [2]. It is not only Rwanda and Uganda that have done this, in Zambia we are also seeing a mad rash for circumcision; in our experience we saw 85 cases in five years before the happening. When the happening started we got 212 requests in one year; a 2.5 fold increase. We have also seen the rise in circumcision in tribes that never circumcised before in fact one such tribe the Bemba were leading in this study surpassing the traditionally circumcising tribes by far. The reasons for requesting circumcision are also different; in the past $54 \%$ of the children were circumcised for traditional reasons at present we found only $17 \%$ requested for the operation for that reason. In the current study $37 \%$ of the children requested for circumcision for social reasons which was the disguised form of a request for circumcision for HIV infection prevention, there was no such request in children in the past. In the present study $44 \%$, compared to $37 \%$ of the children in past, requested circumcision for Phymosis and no parent requested for foreskin dilatation for their children. It must be said however that the adults' reason for requesting circumcision remains the same i.e. social reasons. We know what "social reasons" means now but we are not sure what it meant in the past.

Historically this phenomenon is not new, for example in English-speaking countries Non-religious circumcision once arose in a climate of negative attitudes towards sex, especially concerning masturbation. In 1878 Karen Erickson Paige wrote an article called The Ritual of Circumcision: "In which she stated that there was a rash for male circumcision in the USA. The reason for the surgical removal of the foreskin, or prepuce, was to control 'masturbatory insanity' - the range of mental disorders that people believed were caused by the 'polluting' practice of 'self-abuse[10]. The increased frequency of circumcision demand seems to come on the medical scene from time to time depending on what is in vogue at the particular time, at present it is HIV prevention, one wonders what will come next.

Even the preventive aspect of circumcision is not new: In 1855, the Quaker surgeon, Jonathan Hutchinson, observed that circumcision appeared to protect against syphilis. Although this observation was challenged by his peers, a 2006 systematic review concluded and agreed with him that the evidence "strongly indicates that circumcised men are at lower risk of contracting syphilis.

In our study most patients sited prevention of HIV transmission as the main reason this was disguised as "Social request" $(37 \%$ in children, $53 \%$ in adults and $46 \%$ in all the patients). This was because most of our clients were uncomfortable to say they sought circumcision for HIV prevention. Even the number of reasons or excuses for requesting circumcision increased because people wanted to be circumcised at any cost. There is a strong acceptance of circumcision among our people and this is running across the tribal divide indeed a paradigm shift.

It is not every one who has accepted the current rash for circumcision. Some have criticized this program and have described it as, at best, a not well thought out program. Several editorials from health care professionals including a recent critique from members of the World Health Organization Regional Office for Europe cite that: One of the major criticisms with implementation, aside from the clear ethical problems it presents, is the fact that in the countries where the largest numbers of circumcisions are being done there is a lack of the required medical competency and skills. Moreover, facilities are in 


\section{International Journal of Science and Research (IJSR) \\ ISSN (Online): 2319-7064 \\ Index Copernicus Value (2013): 6.14 | Impact Factor (2015): 6.391}

dangerously short supply [1],[2]. In addition some people are wondering and want to know whether those requesting for circumcision are thinking about circumcision or whether they want to forgo condom use.[5].

This leaves one wondering whether this is a quick fix that may back fire on us such that we may end up in a worse problem from where we started. Are we getting blinded by the "successful" media campaign and are ignoring the danger signs. Already there have been reports of high complication rates observed in both medical and traditional circumcisions in Kenya[3], [11].Where it has been reported by the WHO researchers, that as many as 35\% of males circumcised by traditional practitioners in Kenya's Bungoma district suffered complications such as bleeding, infection, excessive pain and erectile dysfunction from the procedure, Other common adverse effects reported were pain upon urination, incomplete circumcision requiring recircumcision, and laceration, It is also estimated that $6 \%$ of patients had life-long problems as a result Even in public clinics, the complication rate was 18 percent[11].Our complication rate was $8.6 \%$ although these were minor complications except for one patient who had severe wound infection. We do not know the long term effects as at now.

There are many reasons why the complication rate can be high chief among these being lack of resources and training this was confirmed by the study which said many clinicians lacked sharp and clean instruments and few were formally trained.

There is also a lot of confusion when it comes to the benefits of circumcision over and above the preventive aspects: Media hype has pushed circumcision in the minds of our people by amplifying the benefits; researchers like Krieger et al report that adult male circumcision is not associated with sexual dysfunction. In their study, circumcised men reported increased penile sensitivity and enhanced ease of reaching orgasm. They further claim that their data indicate that integration of male circumcision into programs to reduce HIV risk is unlikely to adversely affect male sexual function[5]. However there is a lot of data which indicate quite the opposite: Some reports say nearly all men who were circumcised after infancy reported some sexual dysfunction, erectile problems or premature ejaculation, and one in five reported some complication as a result of the circumcision [4].

Even the preventive role of circumcision is doubted by some Australian reports, they say that circumcision was not a cost effective method for preventing HIV, circumcising all gay men in Australia would cost \$200 million the first two years and only prevent 37 infections over the next 10 years and 57 by 2030[ 6]. David Templeton of the National Centre in HIV Epidemiology and Clinical Research in Sydney noted the following: Only nine percent of all HIV infections overall can be attributed to being uncircumcised, not enough to advocate throwing out condoms or advocating widespread circumcision [6].

Stevie Clayton, chief executive of the AIDS Council of New South Wells, notes that in a public health sense, a mass circumcision program is very unlikely to be an effective or cost-effective way to go [7].
In the light of this information it appears as though we may be sending the wrong message to our people. In our region the population has responded very well to the circumcision drive. The traditional mode in which circumcision was viewed has changed; it certainly has become fashionable to be circumcised among our people today. But we do not know the future implication of all this and one wonders whether this is the right way of spending our meager resources. However the prevention of the spread HIV is being controlled and circumcision is playing a major role. We also must accept that circumcision is also protecting a lot of our women from Cancer of the cervix.

\section{References}

[1] WHO Programmes and projects HIV/AIDS Topics Male circumcision for HIV prevention

[2] WHO Reports www.circumcisionandhiv.com/2007

[3] WHO Reports www.circumcisionandhiv.com/2008

[4] Dennet, Harley. Circumcision's HIV protection bottoms out. The Star Observer. August 14, 2008

[5] Krieger JN, Mehta SD, Bailey RC, Agot K, NdinyaAchola JO, Parker C, Moses S Adult Male Circumcision: Effects on Sexual Function and Sexual Satisfaction in Kisumu, Kenya. J Sex Med. 2008 Aug 28

[6] Temptleton DJ et al. Circumcision status and risk of HIV seroconversion in the HIM cohort of homosexually active men in Sydney. Fourth International AIDS Society Conference on HIV Pathogenesis, Treatment and Prevention, Sydney, 2007.

[7] Procedings: 2007 International AIDS Society conference in Sydney, Australia

[8] www.circumcisionand hiv.com UCSF's HIV InSite deliberately ignores Kenya's high circumcision complication rate. Friday, September 12, 2008

[9] UNAIDS. Communications guidance - Male circumcision \& HIV prevention in eastern and southern Africa

[10] History of male circumcision. Wikipedia, the free encyclopedia

[11] MacInnis L. Circumcision problems impair HIV prevention study: Africa.reuters.com Mon 1 Sep 2008, 14:29 GMT 